\title{
Stationary binary subdivision schemes using radial basis function interpolation
}

\author{
Byung-Gook Lee ${ }^{\mathrm{a}, \star}$, Yeon Ju Lee ${ }^{\mathrm{b}}$ and Jungho Yoon ${ }^{\mathrm{b}, \star \star}$ \\ ${ }^{a}$ Division of Internet Engineering, Dongseo University, Busan, 617-716, S. Korea \\ E-mail: lbg@dongseo.ac.kr \\ ${ }^{\mathrm{b}}$ Department of Mathematics and Institute for Mathematical Sciences, Ewha W. University, Seoul, \\ 120-750, S. Korea \\ E-mail: \{lee08;yoon\}@math.ewha.ac.kr
}

Received 16 April 2003; accepted 1 May 2004

Communicated by R. Schaback

Dedicated to Prof. Charles A. Micchelli on the occasion of his 60th birthday

\begin{abstract}
A new family of interpolatory stationary subdivision schemes is introduced by using radial basis function interpolation. This work extends earlier studies on interpolatory stationary subdivision schemes in two aspects. First, it provides a wider class of interpolatory schemes; each $2 L$-point interpolatory scheme has the freedom of choosing a degree (say, $m$ ) of polynomial reproducing. Depending on the combination $(2 L, m)$, the proposed scheme suggests different subdivision rules. Second, the scheme turns out to be a $2 L$-point interpolatory scheme with a tension parameter. The conditions for convergence and smoothness are also studied.
\end{abstract}

Keywords: subdivision scheme, radial basis function interpolation, multiquadrics.

Mathematics subject classifications (2000): 41A05, 41A25, 41A30, 65D10, 65D17.

\section{Introduction}

Subdivision is an efficient method for constructing curves and surfaces in Computer Aided Geometric Design and Computer Graphics. By this method, at each recursion step, new discrete values on a final grid can be computed by weighted sums of the already existing discrete values. In the limit of the recursive process, data are defined on a dense set of points. Considering these data as function values, under certain conditions, a limit continuous function can then be defined by this process.

^ This work was done as a part of Information \& Communication fundamental Technology Research Program supported by Ministry of the Information \& Communication in Republic of Korea.

$\star \star$ Corresponding author. Supported by the Korea Science and Engineering Foundation grant (KOSEF R06-2002-012-01001). 
Starting with a given set of control points at level 0 with the topology of a regular grid $P^{0}=\left\{p_{n}^{0}: n \in \mathbb{Z}\right\}$, the stationary uniform binary subdivision scheme is a process that recursively defines a sequence of nets of control points $P^{k}=\left\{p_{n}^{k}: n \in \mathbb{Z}\right\}_{k \in \mathbb{Z}_{+}}$by a rule of the form

$$
p_{j}^{k+1}=\sum_{n \in \mathbb{Z}} a_{j-2 n} p_{n}^{k}, \quad k \in \mathbb{Z}_{+},
$$

which is denoted formally by $P^{k}=S^{k} P^{0}$. A point of $P^{k}$ is defined by a finite linear combination of points in $P^{k-1}$, with two different rules, or masks, in the curve case (univariate), and four masks in the surface case (bivariate). In general, for each level $k$, only a finite number of coefficients $a_{n}$ are nonzero so that changes in a control point only effect a limited number of patches. Also, this property clearly facilitates the practical implementation of (1.1). A general discussion of stationary subdivision schemes can be found in the works of Cavaretta et al. [3] and Dyn [6].

Since the present topic is considering schemes where each component of the surface is a scalar function generated by the same subdivision scheme, the analysis of a binary subdivision scheme can be reduced to the scalar case to initial sets of control points. Therefore, starting with values $f^{0}=\left\{f_{n}^{0} \in \mathbb{R}: n \in \mathbb{Z}\right\}$, this study considers scalar sets of control points $f^{k}=\left\{f_{n}^{k} \in \mathbb{R}: n \in \mathbb{Z}\right\}$ generated by the relation

$$
f_{j}^{k+1}=\sum_{n \in \mathbb{Z}} a_{j-2 n} f_{n}^{k}, \quad k \in \mathbb{Z}_{+} .
$$

What follows provides the definition of the convergence of a binary subdivision scheme.

Definition 1.1. A binary subdivision scheme $S$ is termed (uniformly) convergent if for every initial data $f^{0}=\left\{f_{n}^{0} \in \mathbb{R}: n \in \mathbb{Z}\right\}$ there exists a continuous function $f$ on $\mathbb{R}$ such that

$$
\lim _{k \rightarrow \infty} \sup _{n \in \mathbb{Z}}\left|f_{n}^{k}-f\left(2^{-k} n\right)\right|=0,
$$

and $f$ is not identically zero for some initial data $f^{0}$.

Let the function $f$ denoted by $S^{\infty} f$ and called a limit function of $S$ or a function generated by $S$. Also introduced is the notion of the basic limit function of a scheme $S$,

$$
\varphi(x)=S^{\infty}\left\{\delta_{0, n}\right\} .
$$

Then, any limit function of the subdivision process can be expressed as

$$
S^{\infty} f^{0}(x)=\sum_{n \in \mathbb{Z}} f_{n}^{0} \varphi(x-n) .
$$

The subdivision scheme $S$ is called $C^{\gamma}$ if its basic limit function, and hence all functions generated by it, is $C^{\gamma}$. 
A binary subdivision scheme is called interpolatory if $a_{2 n}=\delta_{0, n}, n \in \mathbb{Z}$, since then $P^{k} \subset P^{k+1}$ and therefore $P^{0} \subset P^{k}$ for any $k \in \mathbb{Z}_{+}$. An example of an interpolatory subdivision scheme is the four-point scheme by Dyn et al. [7]:

$$
f_{2 j+1}^{k+1}=\left(\frac{1}{2}+\omega\right)\left(f_{j}^{k}+f_{j+1}^{k}\right)-\omega\left(f_{j-1}^{k}+f_{j+2}^{k}\right) .
$$

It is known that this 4-point scheme generates continuous limit functions for $|\omega|<\frac{1}{2}$, and $C^{1}$ limit functions for $0<\omega<0.154$. With the special choice $\omega=\frac{1}{16}$, this 4 point scheme is exact for cubic polynomials, and it has been generalized to the 6-point scheme (see Dyn's survey paper [6]) and symmetric $2 L$-point schemes by Deslauriers and Dubuc in [4] using polynomial interpolation.

In this paper, we are interested in developing a new family of stationary subdivision schemes obtained by using radial basis function interpolation, which is one of the most well-established methods for data representation problems. Specifically, the insertion rule is obtained by taking the value at the inserted point of radial basis interpolation to the data at $2 L$ symmetric points to the inserted one. This work extends earlier studies on interpolatory subdivision schemes in two aspects. First, it provides a wider class of interpolatory scheme; each $2 L$-point interpolatory scheme has the freedom of choosing a degree (say, $m$ ) of polynomial reproducing. Hence, depending on the combination $(2 L, m)$, the scheme suggests different subdivision rules. Second, the scheme proposed here turns out to be a $2 L$-point interpolatory scheme with a tension parameter. Thus, each aforementioned interpolatory rule can be a special case of the proposed interpolatory scheme. Then, the fundamental questions of interest for the new subdivision scheme are as follows: Does the scheme converge? What is its smoothness? What is its approximation order? Note that for interpolatory subdivision schemes, convergence implies uniform convergence, since the values $\left\{f_{n}^{k}\right\}$ are on the limit function.

The general setting and basic theory of radial basis function interpolation will be discussed in section 2. Among the many radial basis functions, the major concern here is with the basis functions

$$
\phi_{\lambda}(x)=\left(x^{2}+\lambda^{2}\right)^{\beta / 2}, \quad \lambda>0, \beta \in 2 \mathbb{N}-1,
$$

which is the so-called multiquadrics. One of the reasons for choosing this particular function is the desire to use the parameter $\lambda$ as a 'tension' parameter.

The paper is organized as follows: section 2 introduces the general theories related to radial basis function interpolation, then constructs a new class of interpolatory subdivision schemes using radial basis function interpolation. In section 3, we study the convergence and smoothness of the new subdivision scheme, and then find its approximation order.

Throughout this paper, for any positive integer $n, \Pi_{n}$ stands for the subspace $C(\mathbb{R})$ consisting of all algebraic polynomials of degrees less than $n$. All matrices and vectors are written in bold character, for example, $\mathbf{g}$. 


\section{Interpolatory subdivision scheme using radial basis function}

\subsection{Radial basis function interpolation}

Radial basis function interpolation is a very popular and convenient tool for data representation problems. This section introduces the general setting and basic theory of radial basis function interpolation. Suppose that a continuous function $f: \mathbb{R} \rightarrow \mathbb{R}$ is known only at a set of discrete points $X:=\left\{x_{1}, \ldots, x_{N}\right\}$ in $\Omega \subset \mathbb{R}$. The radial basis function interpolation to $f$ on $X$ starts with choosing a basis function $\phi$, and then it defines an interpolant by

$$
S_{f, X}(x):=\sum_{n=1}^{N} \alpha_{n} \phi\left(x-x_{n}\right)+\sum_{i=1}^{m} \beta_{i} p_{i}(x),
$$

where $p_{1}, \ldots, p_{m}$ is a basis for $\Pi_{m}$ and the coefficients $\alpha_{n}$ are chosen so that

$$
\sum_{n=1}^{N} \alpha_{n} p_{i}\left(x_{n}\right)=0, \quad i=1, \ldots, m .
$$

For a wide choice of functions $\phi$ and polynomials in $\Pi_{m}$, including the case $m=1$, the coefficients of $S_{f, X}$ are required to satisfy the $(N+m) \times(N+m)$ system of linear equations, which can be written in a matrix form as

$$
\left(\begin{array}{cc}
\mathbf{A} & \mathbf{P} \\
\mathbf{P}^{\mathrm{T}} & \mathbf{0}
\end{array}\right)\left(\begin{array}{l}
\mathbf{a} \\
\mathbf{b}
\end{array}\right)=\left(\begin{array}{l}
\mathbf{f} \\
\mathbf{0}
\end{array}\right)
$$

where $\mathbf{A}$ and $\mathbf{P}$ are the $N \times N$ and $N \times m$ matrices that have the elements $\mathbf{A}_{i j}=\phi\left(x_{i}-x_{j}\right)$ and $\mathbf{P}_{i j}=p_{j}\left(x_{i}\right)$, respectively. Further, $\mathbf{a} \in \mathbb{R}^{N}$ and $\mathbf{b} \in \mathbb{R}^{m}$ are the vectors of the coefficients of $S_{f, X}$, and the components of $\mathbf{f}$ are the data $f\left(x_{j}\right)$ with $j=1, \ldots, N$. In a fundamental paper by Micchelli [11], the existence and uniqueness of the solution of the linear system (2.2) is ensured when the basis function $\phi$ is a conditionally positive definite function:

Definition 2.1. Let $\phi: \mathbb{R} \rightarrow \mathbb{R}$ be a continuous function. We say that $\phi$ is conditionally positive definite of order $n \in \mathbb{N}:=\{1,2, \ldots\}$ if for every finite set of pairwise distinct points $X=\left\{x_{1}, \ldots, x_{N}\right\} \subset \mathbb{R}$ and for every $\alpha=\left(\alpha_{1}, \ldots, \alpha_{N}\right) \in \mathbb{R}^{N} \backslash 0$ satisfying

$$
\sum_{j=1}^{N} \alpha_{j} p\left(x_{j}\right)=0, \quad p \in \Pi_{n},
$$

the quadric form

$$
\sum_{i=1}^{N} \sum_{j=1}^{N} \alpha_{i} \alpha_{j} \phi\left(x_{i}-x_{j}\right)
$$

is positive definite. 
In particular, the radial basis function interpolant $S_{f, X}$ in (2.1) has a Lagrange-type representation:

$$
S_{f, X}(x):=\sum_{n=1}^{N} u_{n}(x) f\left(x_{n}\right), \quad u_{n}\left(x_{\ell}\right)=\delta_{\ell, n},
$$

where $u_{n}$ are the Lagrange functions from the space $\operatorname{span}\left\{\phi\left(\cdot-x_{1}\right), \ldots, \phi\left(\cdot-x_{N}\right)\right\}+\Pi_{m}$. They satisfy not only the above properties but also reproduce polynomials up to a degree less than $m$, i.e.,

$$
\sum_{j=1}^{N} u_{j}(x) p\left(x_{j}\right)=p(x), \quad p \in \Pi_{m} .
$$

Introducing vectors

$$
\begin{aligned}
& \mathbf{R}(x):=\left(\phi\left(x-x_{1}\right), \ldots, \phi\left(x-x_{N}\right)\right)^{\mathrm{T}}, \\
& \mathbf{S}(x):=\left(p_{1}(x), \ldots, p_{m}(x)\right)^{\mathrm{T}},
\end{aligned}
$$

the vector $\mathbf{u}(x):=\left(u_{1}(x), \ldots, u_{N}(x)\right)^{\mathrm{T}}$ can be obtained by solving the linear system

$$
\left(\begin{array}{cc}
\mathbf{A} & \mathbf{P} \\
\mathbf{P}^{\mathrm{T}} & \mathbf{0}
\end{array}\right)\left(\begin{array}{l}
\mathbf{u}(x) \\
\mathbf{v}(x)
\end{array}\right)=\left(\begin{array}{l}
\mathbf{R} \\
\mathbf{S}
\end{array}\right) \text {. }
$$

A reader interested in knowing more about the state-of-the-art in the area of radial basis function method may find it useful to consult the surveys of Buhmann [2], Dyn [5], and Powell [12]. Another important source is the works of Wu and Schaback [13] and particularly Madych and Nelson [9, 10], who developed a theory of interpolation based on reproducing kernel Hilbert spaces. In addition, approximation orders on Sobolev space by using smooth radial basis functions (e.g., multiquadric) have been studied by Yoon $[14,15]$.

\subsection{Subdivision scheme using radial basis function interpolation}

An interesting class of this study is the family of univariate interpolatory subdivision schemes. Interpolatory subdivision schemes are refinement rules based on symmetric $2 L$-points and defined by interpolating the values at the $2 L$-points via a linear combination of suitable $2 L$ functions. The subdivision schemes considered in this paper are stationary uniform in the sense that they are independent of the positions and levels of refinement. The general form of a $2 L$-point interpolatory subdivision scheme is as follows:

$$
\begin{aligned}
f_{2 j}^{k+1} & =f_{j}^{k}, \\
f_{2 j+1}^{k+1} & =\sum_{n=-L+1}^{L} a_{1-2 n} f_{j+n}^{k}, \quad j \in \mathbb{Z}, k \in \mathbb{Z}_{+} .
\end{aligned}
$$

This condition guarantees that the point set $f^{k}=\left\{f_{n}^{k}: n \in \mathbb{Z}\right\}$ belongs to the limit function. 
Now, we present a stationary $2 L$-point interpolatory subdivision scheme using radial basis function interpolation. Its construction is based on the Lagrange-type representation in (2.3): considering $\bar{f}^{k}$ as the underlying function of the set $\left\{f_{n}^{k}\right\}_{n \in \mathbb{Z}}$, i.e., $\bar{f}^{k}\left(n 2^{-k}\right)=f_{n}^{k}$, on the stage $k$, the value at a point of the finer level $k+1$ is determined by evaluating the value of the radial basis function interpolation (see (2.1)) at the insertion point. More specifically, denoting

$$
X_{k, j}=\left\{(j+\ell) 2^{-k}: \ell=-L+1, \ldots, L\right\}
$$

and invoking the Lagrange-type representation of the radial basis function interpolation in (2.3), the values $f_{2 j+1}^{k+1}$ are defined by

$$
f_{2 j+1}^{k+1}=S_{\bar{f}^{k}, X_{k, j}}\left(2^{-k}\left(j+2^{-1}\right)\right)=\sum_{n=j-L+1}^{j+L} u_{j, n}^{[k]}\left(2^{-k}\left(j+2^{-1}\right)\right) f_{n}^{k},
$$

where $u_{j, n}^{[k]}$ indicates the Lagrange function on $X_{k, j}$. This means that the mask set of the proposed subdivision scheme is defined by

$$
a_{2(j-n)+1}:=u_{j, n}^{[k]}\left(2^{-k}\left(j+2^{-1}\right)\right), \quad j \in \mathbb{Z} .
$$

Here, the mask set $\left\{a_{n}\right\}$ looks like it depends on the position $j$ and level $k$. Yet, it will be seen that it is independent of $j$ and $k$ under certain suitable conditions of the basis function. Further, among many radial basis functions, aiming at the construction of an interpolatory subdivision scheme, the major concern of this study is with the multiquadrics

$$
\phi_{\lambda}(x):=\left(x^{2}+\lambda^{2}\right)^{\beta / 2}, \quad \lambda>0, \beta \in 2 \mathbb{N}-1,
$$

whose properties are quite well understood, both theoretically as well as practically. One of the reasons for choosing this particular function is the desire to use the parameter $\lambda$ as a 'tension' parameter. In particular, for the purpose of making a stationary subdivision scheme, the tension parameter $\lambda$ is dilated depending on the level $k$. That is, for each level $k, \phi_{\lambda_{k}}(x):=\left(x^{2}+\lambda_{k}^{2}\right)^{\beta / 2}$ is employed with

$$
\lambda_{k}=2^{-k} \lambda .
$$

The following theorem proves that the mask set $\left\{a_{n}\right\}$ is independent of the location $j$ and level $k$, which means the binary subdivision scheme (henceforth, called $S_{a}$ ) associated with this rule is said to be stationary and uniform.

Theorem 2.2. Let $\phi_{\lambda}(x)=\left(x^{2}+\lambda^{2}\right)^{\beta / 2}$. Suppose that the mask set $\left\{a_{n}\right\}$ is given by the Lagrange type representation of $\phi_{\lambda}$-interpolation as in (2.8). Assume that for each level $k$, the basis function $\phi_{\lambda_{k}}$ is employed with $\lambda_{k}=2^{-k} \lambda$. Then, the subdivision scheme $S_{a}$ associated with the mask set $\left\{a_{n}\right\}$ is stationary, that is, the mask set is independent of the positions and levels. 
Proof. Let $j \in \mathbb{Z}$. Then, since each element $x_{\ell} \in X_{j}$ has the form $x_{\ell}:=2^{-k}(j+\ell)$ with $\ell=-L+1, \ldots, L$, we can write

$$
\phi_{\lambda_{k}}\left(x_{i}-x_{\ell}\right)=\phi_{\lambda_{k}}\left(2^{-k}(i-\ell)\right)=2^{-\beta k} \phi_{\lambda}(i-\ell),
$$

where the second equality is immediate by the fact $\lambda_{k}=2^{-k} \lambda$. Therefore, invoking (2.6) and choosing $p_{n}(x)=2^{-k \beta}\left(2^{k} x-j\right)^{n-1}, n=1, \ldots, m$, as a basis $\Pi_{m}$, the vector of the mask set $\mathbf{u}:=\left\{a_{2(j-n)+1}: n=-L+1, \ldots, L\right\}$ (see (2.8)) can be obtained by solving the following linear system

$$
\left(\begin{array}{cc}
\overline{\mathbf{A}} & \overline{\mathbf{P}} \\
\overline{\mathbf{P}}^{\mathrm{T}} & \mathbf{0}
\end{array}\right)\left(\begin{array}{l}
\mathbf{u} \\
\mathbf{v}
\end{array}\right)=\left(\begin{array}{l}
\overline{\mathbf{R}} \\
\overline{\mathbf{S}}
\end{array}\right)
$$

where the matrices $\overline{\mathbf{A}}, \overline{\mathbf{P}}, \overline{\mathbf{R}}$, and $\overline{\mathbf{S}}$ have elements of the form

$$
\begin{array}{ll}
\overline{\mathbf{A}}(i, \ell):=\phi_{\lambda}(i-\ell), & \overline{\mathbf{P}}(\ell, n):=\ell^{n-1}, \\
\overline{\mathbf{R}}(\ell):=\phi_{\lambda}\left(2^{-1}-\ell\right), & \overline{\mathbf{S}}(\ell):=2^{-\ell+1} .
\end{array}
$$

It is clear from the system (2.9) that $\mathbf{u}$ is independent of the position $j$ and level $k$. Hence, the proposed scheme $S_{a}$ is stationary uniform.

Due to the above theorem, recalling (2.8), the definition of the masks $a_{1-2 n}$ can be induced as follows:

$$
a_{1-2 n}:=u_{n}\left(2^{-1}\right), \quad n=-L+1, \ldots, L,
$$

where $u_{n}(x)$ is the Lagrange function on $X_{0}=\{-L+1, \ldots, L\}$, that is,

$$
u_{n}(x) \in \operatorname{span}\left\{\phi_{\lambda}(x-\ell): \ell \in X_{0}\right\}+\Pi_{m} .
$$

It is also of interest to point out that besides the polynomial reproducing property of $a_{1-2 n}$ in (2.4), the mask set $\left\{a_{n}\right\}$ reproduces the functions $\phi_{\lambda}(\cdot-\ell)$ with $\ell \in X_{0}$ in the sense that

$$
\sum_{n \in \mathbb{Z}} a_{1-2 n} \phi_{\lambda}(n-\ell)=\phi_{\lambda}\left(2^{-1}-\ell\right) .
$$

Table 1 provides the general form of the mask set $\left\{a_{1-2 n}\right\}, n=-L+1, \ldots, L$, of $2 L$-point interpolatory subdivision scheme $S_{a}$ for the case $m>2(L-2)$. For a chosen basis function $\phi_{\lambda}(x)=\left(x^{2}+\lambda^{2}\right)^{\beta / 2}$, the parameter $w$ in table 1 is given in terms of $\lambda$, i.e., $w=w(\lambda)$. But, since the explicit forms of $w(\lambda)$ are too long to provide here (see the appendix for an example), the relations between $w(\lambda)$ and $\lambda$ are described via graphs in figure 1 for $\lambda \in[0,30]$. It is observed from figure 1 that for all $\lambda \in[0,30]$, each $w(\lambda)$ belongs to the range in the above table 1 . Also as $L$ increases, the ranges of $w$ in table 1 become narrower. It is also interesting to point out that for each pair $m=2 n-1,2 n$ with $1 \leqslant n \leqslant L$, the same mask set is obtained. It would seem that this is because of the symmetric properties of the mask set. Further, when $m>2(L-1)$, this scheme is identical to Deslauriers and Dubuc's $2 L$-point interpolatory scheme. The 4- and 6-point rules in [6] are special cases of the proposed scheme with $m=1,3$ and $2 L=4,6$, 
Table 1

General forms of the mask sets $\left\{a_{1-2 n}\right\}$ with $n=-L+1, \ldots, L$ of $2 L$-point interpolatory subdivision scheme $S_{a}$ using $\phi_{\lambda}$. This is for the case that $m>2(L-2)$. Here, $m$ will be used as the smoothing factor (see (3.3)). By computing $\left\|\left((1 / 2) S_{L}\right)^{10}\right\|_{\infty}<1$ with MAPLE 8 , digits $=30$, the above ranges of $w$ are obtained.

\begin{tabular}{cccc}
\hline $2 L$ & Mask & Range of $w$ & Smoothness \\
\hline $4 \quad\left[-w, \frac{1}{2}+w, \frac{1}{2}+w,-w\right]$ & $0<w<0.183$ & $C^{1}$ \\
$6 \quad\left[w,-3 w-\frac{1}{16}, 2 w+\frac{9}{16}, 2 w+\frac{9}{16},-3 w-\frac{1}{16}, w\right]$ & $0<w<0.042$ & $C^{2}$ \\
$8 \quad\left[-w, 5 w+\frac{3}{256},-9 w-\frac{25}{256}, 5 w+\frac{75}{128}\right.$, & $0.0016<w<0.0084$ & $C^{3}$ \\
& $\left.\quad 5 w+\frac{75}{128},-9 w-\frac{25}{256}, 5 w+\frac{3}{256},-w\right]$ & \\
$10 \quad\left[w,-7 w-\frac{5}{2048}, 20 w+\frac{49}{2048},-28 w-\frac{245}{2048}\right.$, & $0.0005<w<0.0016$ & $C^{4}$ \\
& $\quad 14 w+\frac{1225}{2048}, 14 w+\frac{1225}{2048}$, & & \\
& $\left.-28 w-\frac{245}{2048}, 20 w+\frac{49}{2048},-7 w-\frac{5}{2048}, w\right]$ & & \\
\end{tabular}

respectively. Note that for these 4 - and 6-point schemes, table 1 provides wider ranges of $0<w<0.183$ and $0<w<0.042$ than the known results of $0<w<0.154$ and $0<w<0.02$ in [6], respectively. Finally, it should be remarked that $\beta$ in $\phi_{\lambda}$ has no effect on either the smoothness of $S_{a}$ or the general form of $a_{1-2 n}$. Usually, $\beta$ and $m$ are chosen to be $\beta \geqslant m$.

Remark. From a practical point of view, the tension parameter $\lambda>0$ in (3.4) is supposed to be a rather small number. Indeed, if $\lambda$ is large, the linear system becomes numerically highly unstable (see [1]). However, one interesting question arising with our scheme $S_{a}$ using $\phi_{\lambda}$-interpolation is whether the scheme $S_{a}$ converges as $\lambda$ tends to $\infty$. Our observation is that as $\lambda$ tends to $\infty$, the mask set $\left\{a_{n}\right\}$ asymptotically approaches the mask set of Deslauriers and Dubuc's scheme. For instance, the 4-point scheme $S_{a}$ with $\beta=1$ and $m=1$ has the tension parameter $w(\lambda)$ in the mask set $\mathbf{a}(\lambda)=\left\{a_{1-2 n}\right\}=\left\{-w(\lambda), \frac{1}{2}+w(\lambda), \frac{1}{2}+w(\lambda),-w(\lambda)\right\}$, as in table 1. From figure 1 , it is seen that as $\lambda \rightarrow \infty, w(\lambda)$ converges to $16^{-1}$. That is, $\mathbf{a}(\lambda)$ converges to the mask set of Deslauriers and Dubuc's 4-point scheme (see also (1.3)). Similarly, for the 6-, 8- and 10-point schemes, it can be seen from figure 1 that as $\lambda \rightarrow \infty, w(\lambda)$ converges to $3 / 256,5 / 2048$ and $35 / 65536$, respectively, which constitute the mask sets of Deslauriers-Dubuc's interpolatory schemes. Actually, these convergence properties may be proved in the sense of the asymptotical equivalent relation between (nonstationary) subdivision schemes, yet this is not the concern of the current paper. A detailed analysis on nonstationary schemes is presented in the manuscript [8]. 


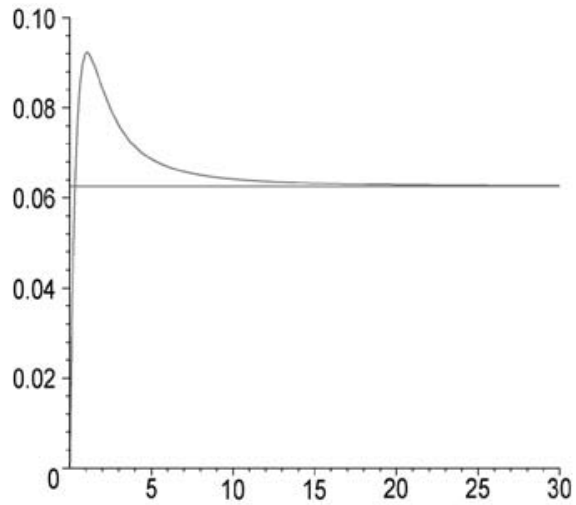

(A) 4-point scheme

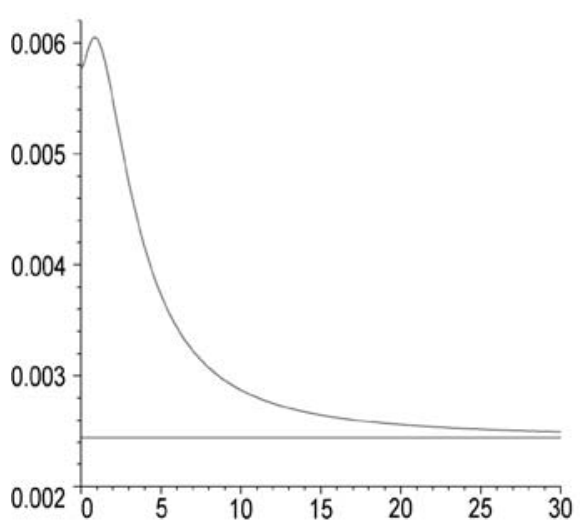

(C) 8-point scheme

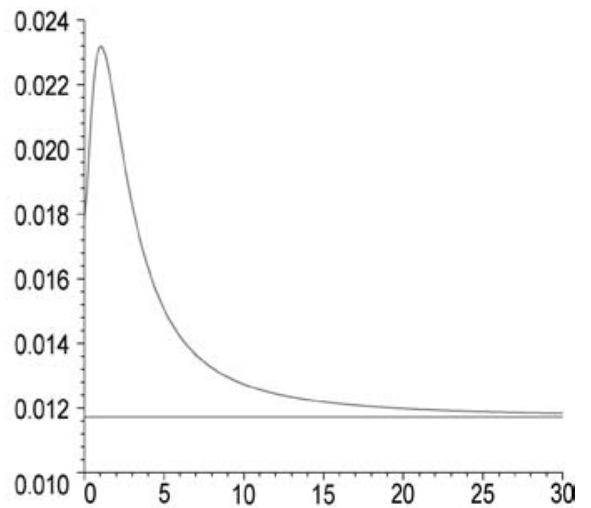

(B) 6-point scheme

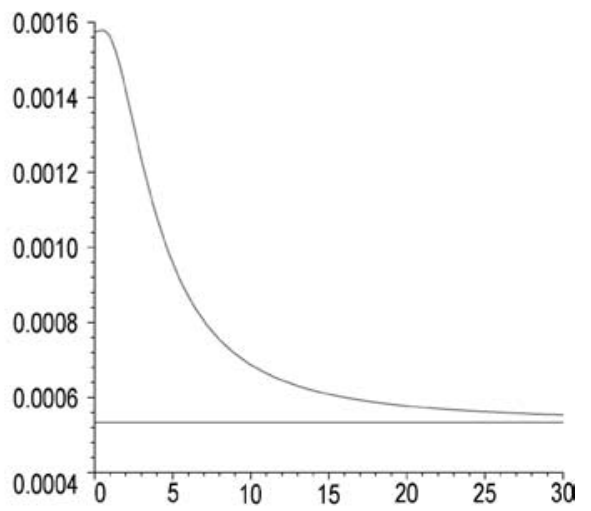

(D) 10-point scheme

Figure 1. Graphs of the tension parameters $w(\lambda)$ in the mask sets $\left\{a_{1-2 n}\right\}$ in table 1. For the basis function $\phi_{\lambda}(x)=\left(x^{2}+\lambda\right)^{\beta / 2}$ and smoothing factor $m$, we used $(\beta, m)=(1,1),(3,3),(5,5)$ and $(7,7)$. The reference lines indicate $\omega=1 / 16,3 / 256,5 / 2048,35 / 65536$ which constitute the mask sets of $2 L$-point Deslauriers and Dubuc's scheme for $2 L=4,6,8$ and 10, respectively.

Now, with the mask set $\left\{a_{n}\right\}$ at hand, the fundamental questions related to this scheme are as follows: Does the scheme converge? What is its smoothness? What is the approximation order of $S_{a}$ ? The following sections discuss these questions. Note that for interpolatory subdivision schemes, convergence implies uniform convergence, since the values $f_{n}:=\left\{f_{n}^{k}: n \in \mathbb{Z}\right\}$ are on the limit function.

\section{Smoothness analysis}

The goal of this section is to show the convergence and smoothness of the interpolatory binary subdivision scheme $S_{a}$ constructed in section 2.2. A necessary condition 
for the uniform convergence of an interpolatory subdivision scheme associated with the mask set $\left\{a_{n}: n \in \mathbb{Z}\right\}$ is

$$
\sum_{n \in \mathbb{Z}} a_{1-2 n}=1
$$

Here, one should note that $a_{2 n}=\delta_{0, n}$. In fact, invoking the definition $a_{1-2 n}=u_{n}\left(2^{-1}\right)$ with the Lagrange function $u_{n}$ (see (2.9)), this condition is an immediate consequence of the polynomial reproducing property of $u_{n}$ in the sense of (2.4).

To simplify the presentation of a subdivision scheme and its analysis, it is convenient to assign to each rule, defined by a set of masks $a_{n}$, the Laurent polynomial

$$
a(z):=\sum_{n \in \mathbb{Z}} a_{n} z^{n} .
$$

The fact that only a finite number of coefficients $a_{n}$ are nonzero implies that the Laurent polynomial $a(z)$ has a finite degree. Further, the Laurent polynomial corresponding to the iterated scheme $\left(S_{a}\right)^{\ell}, \ell>0$, is given by

$$
a^{[\ell]}(z)=\prod_{j=0}^{\ell-1} a\left(z^{2^{j}}\right)=\sum_{n \in \mathbb{Z}} a_{n}^{[\ell]} z^{n},
$$

where the scheme corresponding to $\left\{a_{n}^{[\ell]}\right\}$ is a $2^{\ell}$ different rule mapping $f^{k}$ to $f^{k+\ell}$, that is,

$$
f_{n+2^{\ell} \alpha}^{k+\ell}=\sum_{\beta \in \mathbb{Z}} a_{n+2^{\ell} \beta}^{[\ell]} f_{\alpha-\beta}^{k}, \quad n=0,1, \ldots, 2^{\ell}-1 .
$$

With the above setting, the main tool for the analysis of convergence of a stationary subdivision scheme is given as follows:

Theorem 3.1 [6]. Let $S_{1}$ be a subdivision scheme with the property

$$
d f^{k}=S_{1} d f^{k-1}
$$

where $f^{k}=S^{k} f^{0}$ and $\left(d f^{k}\right)_{\alpha}=2^{k}\left(f_{\alpha}^{k}-f_{\alpha-1}^{k}\right)$. Then a stationary subdivision scheme $S$ is a uniformly convergent if and only if the scheme $\frac{1}{2} S_{1}$ converges uniformly to the zero function for all initial data $f^{0}$.

For the existence of the subdivision scheme $S_{1}$, and for more details about the above discussion, the readers are referred to the paper [6]. In the following theorem, a sufficient condition for $S_{a}$ to be in $C^{\gamma}, \gamma \geqslant 1$ is provided.

Theorem 3.2 (Smoothness of stationary subdivision scheme [6]). Consider a stationary binary subdivision scheme $S_{a}$ with the characteristic Laurent polynomial

$$
a(z)=\frac{1}{2}(1+z) a_{1}(z) .
$$


If $S_{a_{1}}$ corresponding to the Laurent polynomial $a_{1}(z)$ is $C^{\gamma}$, the scheme $S_{a}$ is a convergent, and the basic limit function $\varphi$ of $S_{a}$ is in $C^{\gamma+1}(\mathbb{R})$.

Checking if $S_{a}$ is $C^{1}$ is reduced to verifying that $S_{a_{1}}$ is $C^{0}$. It says that to assure a certain smoothness of the scheme $S_{a}$, the Laurent polynomial should have the factor $2^{-\ell}(1+z)^{\ell}$ with $\ell \in \mathbb{N}$, which directly depends on the order of the polynomial reproducing property of the mask set. Recalling the definition of radial basis function interpolation in (2.1), we find that $S_{f, X}$ reproduces polynomials up to degree $m-1$, i.e., $S_{p, X}(x)=p(x)$ for any $p \in \Pi_{m}$. Then, the Laurent polynomial $a(z)$ associated to the scheme $S_{a}$ by radial basis function interpolation has the form:

Proposition 3.3. Let $S_{a}$ be a $2 L$-point interpolatory subdivision scheme using radial basis function interpolation $S_{f, X}$ as in (2.1). Assume that $S_{p, X}(x)=p(x)$ for any $p \in$ $\Pi_{m}$, and let $\left\{a_{n}\right\}$ be the mask set $S_{a}$. Then the Laurent polynomial $a(z)$ associated to $S_{a}$ is given by

$$
a(z)=2^{-m}(1+z)^{m} q(z)
$$

with a suitable Laurent polynomial $q(z)$.

Proof. It is sufficient to prove that $a^{(\ell)}(-1)=0$ with $\ell<m$. From (3.1), we find that this is equivalent to showing that

$$
\sum_{n \in \mathbb{Z}}(-1)^{n} n^{\ell} a_{n}=0
$$

For this proof, observe that

$$
\begin{aligned}
\sum_{n \in \mathbb{Z}}(-1)^{n} n^{\ell} a_{n} & =\sum_{n \in \mathbb{Z}}(2 n)^{\ell} a_{2 n}-\sum_{n \in \mathbb{Z}}(2 n+1)^{\ell} a_{2 n+1} \\
& =\delta_{0, \ell}-\sum_{n \in \mathbb{Z}}(1-2 n)^{\ell} a_{1-2 n} .
\end{aligned}
$$

Applying the polynomial reproducing property of $\left\{a_{1-2 n}\right\}$ (see (2.4)), it is easy to obtain the identity $\sum_{n \in \mathbb{Z}}(1-2 n)^{\ell} a_{1-2 n}=\delta_{0, \ell}$. Hence, (3.3) is concluded.

The norm of the subdivision scheme $S_{a}$ is given by

$$
\left\|S_{a}\right\|_{\infty}=\max \left\{\sum_{\alpha \in \mathbb{Z}}\left|a_{2 \alpha}\right|, \sum_{\alpha \in \mathbb{Z}}\left|a_{2 \alpha+1}\right|\right\} .
$$

Moreover, invoking (3.2), the norm of the iterated scheme $S_{a}^{\ell}$ is given by

$$
\left\|S_{a}^{\ell}\right\|_{\infty}=\max \left\{\sum_{\alpha \in \mathbb{Z}}\left|a_{\gamma+2^{\ell} \alpha}^{[\ell]}\right|: \gamma=0, \ldots, 2^{\ell}-1\right\} .
$$

Then, according to theorem 3.1, the proof of convergence (further smoothness) of the subdivision scheme $S_{a}$ can be done by the following two steps: first, derive the mask set 
of the scheme $\frac{1}{2} S_{1}$, and second, compute $\left\|\left(\frac{1}{2} S_{1}\right)^{k}\right\|_{\infty}$ to show the existence of an $M \in \mathbb{N}$ such that $\left\|\left(\frac{1}{2} S_{1}\right)^{M}\right\|_{\infty}<1$. If $S_{a}$ converges, such a number $M$ exists. In fact, the Laurent polynomial corresponding to $S_{1}$ is denoted by

$$
a_{1}(z):=\frac{2 z}{1+z} a(z) .
$$

Based on establishing the uniform convergence of the scheme $S_{a}$, the next issue is proving the smoothness of $S_{a}$. Using theorem 3.2, we consider the scheme $S_{2}$ associated to the Laurent polynomial

$$
a_{2}(z):=\frac{2 z}{1+z} a_{1}(z)
$$

and compute $\left\|\left(\frac{1}{2} S_{2}\right)^{k}\right\|_{\infty}$ to find an $M \in \mathbb{N}$ such that $\left\|\left(\frac{1}{2} S_{2}\right)^{M}\right\|_{\infty}<1$. By continuing this process, we can find the smoothness of the given scheme $S_{a}$.

The remainder of this section is devoted to proving the smoothness of the $2 L$-point interpolatory scheme $S_{a}$ by computing $\left\|\left(\frac{1}{2} S_{\ell}\right)^{k}\right\|_{\infty}$ with $\ell=1,2, \ldots$ As observed in section 2.2, the masks $a_{1-2 n}$ associated to the scheme $S_{a}$ are obtained by evaluating the Lagrange function $u_{n}\left(2^{-1}\right)$ where

$$
u_{n}(x) \in \operatorname{span}\left\{\phi_{\lambda}(x+L-1), \ldots, \phi_{\lambda}(x-L)\right\}+\Pi_{m},
$$

with

$$
\phi_{\lambda}(x)=\left(x^{2}+\lambda\right)^{\beta / 2} .
$$

Here, depending on the choice of $\lambda$ and $\beta$, we provide various subdivision rules, i.e., the masks $a_{1-2 n}$. Furthermore, for each $2 L$-point subdivision rule $S_{a}$, there is a freedom

Table 2

Smoothness of $2 L$-point interpolatory subdivision scheme using the multiquadric function $\phi_{\lambda}(x)=\left(x^{2}+\lambda^{2}\right)^{\beta / 2}$ with $\beta \in 2 \mathbb{N}-1$. For each chosen $\beta$ and smoothing factor $m$ in (3.3), the corresponding smoothness is given. When $m>2(L-1)$, this scheme is identical to Deslauriers-Dubuc's $2 L$-point interpolatory scheme.

\begin{tabular}{cl}
\hline $2 L$ & \multicolumn{1}{c}{$(\beta ; m):$ smoothness } \\
\hline 4 & $(m=1, \ldots, 4 ; \beta=1,3): C^{1}$ limit function \\
6 & $(m=1,2 ; \beta=1,3): C^{1}$ limit function \\
& $(m=3, \ldots, 6 ; 3 \leqslant \beta \leqslant 2 m-1): C^{2}$ limit function \\
8 & $(m=1,2 ; \beta=1,3): C^{1}$ limit function \\
& $(m=3,4 ; 3 \leqslant \beta \leqslant 2 m-1): C^{2}$ limit function \\
& $(m=5, \ldots, 8 ; 5 \leqslant \beta \leqslant 2 m-1): C^{3}$ limit function \\
10 & $(m=1,2 ; \beta=1,3): C^{1}$ limit function \\
& $(m=3,4 ; 3 \leqslant \beta \leqslant 2 m-1): C^{2}$ limit function \\
& $(m=5,6 ; 5 \leqslant \beta \leqslant 2 m-1): C^{3}$ limit function \\
& $(m=7, \ldots, 10 ; 7 \leqslant \beta \leqslant 2 m-1): C^{4}$ limit function \\
\hline
\end{tabular}


of the degree $m$ of polynomial reproducing. When considering all these ingredients, it is basically almost impossible to analyze the smoothness of $S_{a}$ without the help of a computer program. Thus, the MAPLE program is utilized to figure out the convergence and smoothness of $S_{a}$ with different inputs of $2 L, \lambda$ and $m$. In particular, we could get the smoothness as in table 2 for $\lambda \in[0,30]$ with $2 L=4, \ldots, 10$. Further, it is necessary to point out that the basis function $\phi_{\lambda}$ is conditionally positive definite of order $(\beta+1) / 2$, which requires the condition $m \geqslant(\beta+1) / 2$. Computing

$$
\left\|\left(\frac{1}{2} S_{L}\right)^{10}\right\|_{\infty}<1,
$$

table 2 displays the smoothness of the $2 L$-point scheme $S_{a}$. When $m>2(L-2)$, as observed in table 2 , the $2 L$-point scheme provides the possible highest smoothness. Finally, there is no restriction on the choice of $\beta$, yet $\beta$ and $m$ are normally chosen to be $m \leqslant \beta$. Also, we remark that $\beta$ has no effect on either the smoothness of $S_{a}$ or to the general form of $a_{1-2 n}$.

\section{Approximation order}

Suppose that the given initial data is of the form $f^{0}:=\left\{f_{n}^{0}=f(h n): n \in \mathbb{Z}\right\}$, $h>0$, with an underlying function $f$. Then the limit of a subdivision scheme, say $f^{\infty}$, approximates the sampled function $f$ if $f$ is smooth enough. It is basic to expect that $f^{\infty}$ approximates $f$ better as $h$ tends to 0 . The quality of approximation is improved with the reduction of the density of sampling. Asymptotic approximation powers are usually quantified by the notion of approximation order in terms of $h$. In particular, this study is interested in approximating functions $f$ in a homogeneous Sobolev space. For any $\gamma \in \mathbb{Z}_{+}$, the homogeneous Sobolev space $W_{\infty}^{\gamma}(\mathbb{R})$ consists of all functions $g$ for which $g^{(\gamma)} \in L_{\infty}(\mathbb{R})$.

Definition 4.1. A binary subdivision scheme $S$ has the approximation order $\gamma$ on a compact set $K \subset \mathbb{R}$ if for a given initial data $f^{0}:=\left\{f_{n}^{0}=f(h n): n \in \mathbb{Z}\right\}$ with a smooth function $f$,

$$
\left|f^{\infty}(x)-f(x)\right| \leqslant C h^{\gamma}, \quad x \in K,
$$

where the constant $C$ is independent of $x$ and $h$, but may depend on $f$.

For the given initial data $f^{0}:=\left\{\delta_{0, n}: n \in \mathbb{Z}\right\}$, the basic limit function of the uniform subdivision scheme $S_{a}$ is defined by

$$
\varphi=\lim _{k \rightarrow \infty} S_{a}^{k}\left\{\delta_{0, n}\right\}
$$

By definition, the basic limit function of a scheme has compact support if the mask of the scheme is of finite support. Denoting $\mathbf{a}=\left\{a_{n}: n \in \mathbb{Z}\right\}$, it is known that $\operatorname{supp}(\varphi)=$ $\operatorname{supp}(\mathbf{a})$. The scheme $S_{a}$ is called $C^{m}$ if its basic limit function is, and hence all functions 
generated by the scheme, are $C^{m}$. From now on, for convenience, we assume that the given initial data is of the form $f^{0}:=\left\{f_{n}^{0}=f\left(2^{-k} n\right): n \in \mathbb{Z}\right\}$ for some $k \in \mathbb{Z}_{+}$with a smooth function $f$. Then, the limit function $f^{\infty}$ can be expressed as

$$
f^{\infty}(x)=\sum_{n \in \mathbb{Z}} f\left(n 2^{-k}\right) \varphi\left(2^{k} x-n\right) .
$$

In what follows, we verify that the approximation order of a subdivision scheme directly depends on the polynomial reproducing property of the basic limit function.

Lemma 4.2. Let $S_{a}$ be the $2 L$-point interpolatory subdivision scheme using radial basis function interpolation $S_{f, X}$ as in (2.1). Assume that $S_{p, X}(x)=p(x)$ for any $p \in \Pi_{m}$, and let $\varphi$ be the basic limit function of $S_{a}$. Then, the map $f \mapsto \sum_{n \in \mathbb{Z}} \varphi\left(2^{k} x-n\right) f\left(2^{-k} n\right)$ reproduces $\Pi_{m}$, i.e.,

$$
\sum_{n \in \mathbb{Z}} \varphi\left(2^{k} x-n\right) p\left(2^{-k} n\right)=p(x), \quad \forall p \in \Pi_{m}
$$

Proof. Let $p$ be a polynomial in $\Pi_{m}$, and assume that the initial data is given by $f_{n}^{0}=$ $p\left(2^{-k} n\right)$. Then, invoking the relation that $a_{1-2 n}=u_{n}\left(2^{-k-1}\right)$ with the Lagrange function $u_{n}$ on $X=\left\{\ell 2^{-k}: \ell=-L+1, \ldots, L\right\}$, the polynomial reproducing property in (2.4) is applied to get

$$
f_{2 j+1}^{1}=\sum_{n \in \mathbb{Z}} a_{1-2 n} f_{j+n}^{0}=\sum_{n \in \mathbb{Z}} u_{n}\left(2^{-k-1}\right) p\left(2^{-k}(j+n)\right)=p\left(2^{-k}\left(j+2^{-1}\right)\right) .
$$

Repeating this process, we find that $S^{\infty} f^{0}(x)=p(x)$ with $f_{n}^{0}=p\left(2^{-k} n\right)$. On the other hand, since $S^{\infty} f^{0}(x)=\sum_{n \in \mathbb{Z}} \varphi\left(2^{k} x-n\right) f_{n}^{0}$, the lemma's claim can be obtained.

We are now ready to prove the approximation order of our subdivision scheme $S_{a}$.

Theorem 4.3. Let $S_{a}$ be a $2 L$-point interpolatory subdivision scheme using radial basis function interpolation $S_{f, X}$ in (2.1). Assume that $S_{p, X}(x)=p(x)$ for any $p \in \Pi_{m}$, and let the given initial data be of the form $f^{0}:=\left\{f_{n}^{0}=f\left(2^{-k} n\right): n \in \mathbb{Z}\right\}$ with a smooth function $f$. If $f \in W_{\infty}^{m}(K)$ with a compact set $K$, then the scheme $S_{a}$ has the approximation order $m$ on $K$.

Proof. First, recalling lemma 4.2, apply the identity $\sum_{\ell \in \mathbb{Z}} \varphi\left(2^{k} x-n\right)=1$ to get the relation

$$
\begin{aligned}
f(x)-f^{\infty}(x) & =f(x)-\sum_{n \in \mathbb{Z}} \varphi\left(2^{k} x-n\right) f\left(n 2^{-k}\right) \\
& =\sum_{n \in \mathbb{Z}} \varphi\left(2^{k} x-n\right)\left(f(x)-f\left(n 2^{-k}\right)\right) .
\end{aligned}
$$


Then, taking Taylor polynomial of $f\left(n 2^{-k}\right)$ of degree $m-1$ at $x$ implies that

$$
\begin{aligned}
f(x)-f^{\infty}(x)= & \sum_{n \in \mathbb{Z}} \varphi\left(2^{k} x-n\right) \sum_{\ell=1}^{m-1} \frac{\left(n 2^{-k}-x\right)^{\ell} f^{(\ell)}(x)}{\ell !} \\
& +\sum_{n \in \mathbb{Z}} \frac{\varphi\left(2^{k} x-n\right)\left(n 2^{-k}-x\right)^{m} f^{(m)}(\xi)}{m !},
\end{aligned}
$$

with $\xi$ between $x$ and $n 2^{-k}$. Here, due to lemma 4.2, the first term on the right-hand side of the above equation is identically zero. Thus, it follows that

$$
\left|f(x)-f^{\infty}(x)\right| \leqslant c 2^{-m k}\left\|f^{(m)}\right\|_{\infty} \sum_{n \in \mathbb{Z}}\left|\varphi\left(2^{k} x-n\right)\left(2^{k} x-n\right)^{m}\right| .
$$

For any $x \in K$, define a set $\Omega_{x}$ by $\Omega_{x}:=\left\{n \in \mathbb{Z}: \varphi\left(2^{k} x-n\right) \neq 0\right\}$. Since $\varphi$ is compactly supported, it is obvious that $\# \Omega_{x} \leqslant C_{\varphi}$ for some constant $C_{\varphi}$. Therefore, we get the bound

$$
\sum_{n \in \mathbb{Z}}\left|\varphi\left(2^{k} x-n\right)\left(2^{k} x-n\right)^{m}\right|=\sum_{n \in \Omega_{x}}\left|\varphi\left(2^{k} x-n\right)\left(2^{k} x-n\right)^{m}\right| \leqslant C_{\varphi, m},
$$

with a constant $C_{\varphi, m}>0$. When combined with (4.3), this theorem is proved.

Remark. Although this study concentrated on the interpolatory binary subdivision schemes using the multiquadrics, the proposed approach can also be applied to any basis function $\phi$ whose Fourier transform $\hat{\phi}$ coincides on $\mathbb{R} \backslash 0$ with some continuous function while having a certain type of singularity (necessarily of a finite order) at the origin, i.e., $\hat{\phi}$ is of the form $\left.|\cdot|\right|^{n} \hat{\phi}=F>0$ with $n \geqslant 0$ and $F \in L_{\infty}(\mathbb{R})$. For instance, the Gaussian function $\phi(x):=\mathrm{e}^{-c x^{2}}, c>0$, and inverse multiquadric function $\phi(x):=\left(x^{2}+\lambda^{2}\right)^{-1 / 2}$, $\lambda>0$, can be candidates.

\section{Appendix}

For a given basis function $\phi_{\lambda}$, the general form of the mask set $\left\{a_{1-2 n}\right\}$ of the $2 L$-point interpolatory scheme can be given in terms of $w=w(\lambda)$. However, the explicit forms of $w(\lambda)$ are too lengthy. For instance, in the case of the 4-point interpolatory scheme using the multiquadrics $\phi_{\lambda}(x)=\left(x^{2}+\lambda^{2}\right)^{1 / 2}$ its mask set is known as

$$
a_{-3}=a_{3}=-w(\lambda), \quad a_{-1}=a_{1}=\frac{1}{2}+w(\lambda),
$$

and with the help of symbolic computation via MAPLE $8, w(\lambda)$ can be written as follows:

$$
\begin{aligned}
w(\lambda)= & -\frac{1}{4}\left(-11 \lambda^{2}+20 \sqrt{1+\lambda^{2}} \lambda+4 \lambda \sqrt{4+\lambda^{2}}+21+7 \sqrt{1+\lambda^{2}} \sqrt{9+\lambda^{2}}\right. \\
& \left.-12 \sqrt{1+\lambda^{2}} \sqrt{4+\lambda^{2}}-4 \sqrt{4+\lambda^{2}} \sqrt{9+\lambda^{2}}-4 \lambda \sqrt{9+\lambda^{2}}\right)^{-1}
\end{aligned}
$$




$$
\begin{aligned}
& \times\left(15 \sqrt{9+4 \lambda^{2}} \sqrt{1+\lambda^{2}}-10 \sqrt{9+4 \lambda^{2}} \lambda-6 \sqrt{9+4 \lambda^{2}} \sqrt{4+\lambda^{2}}\right. \\
& -15 \sqrt{1+4 \lambda^{2}} \sqrt{1+\lambda^{2}}+10 \sqrt{1+4 \lambda^{2}} \lambda-\sqrt{1+4 \lambda^{2}} \sqrt{9+\lambda^{2}} \\
& +6 \sqrt{1+4 \lambda^{2}} \sqrt{4+\lambda^{2}}+\sqrt{9+4 \lambda^{2}} \sqrt{9+\lambda^{2}}+24-4 \lambda^{2}+15 \sqrt{1+\lambda^{2}} \lambda \\
& \left.-15 \sqrt{1+\lambda^{2}} \sqrt{4+\lambda^{2}}+4 \lambda \sqrt{4+\lambda^{2}}+\lambda \sqrt{9+\lambda^{2}}-\sqrt{4+\lambda^{2}} \sqrt{9+\lambda^{2}}\right) .
\end{aligned}
$$

\section{Acknowledgements}

The authors are grateful to the anonymous referee for their valuable suggestions on this paper.

\section{References}

[1] B.J.C. Baxter, N. Sivakumar and J.D. Ward, Regarding the $p$-norms of radial basis interpolation matrices, Constr. Approx. 10 (1994) 451-468.

[2] M.D. Buhmann, New developments in the theory of radial basis function interpolation, in: Multivariate Approximation: From CAGD to Wavelets, eds. K. Jetter and F.I. Utreras (World Scientific, Singapore, 1993) pp. 35-75.

[3] A. Cavaretta, W. Dahmen and C.A. Micchelli, Stationary subdivision, Mem. Amer. Math. Soc. 93 (1991) 1-186.

[4] G. Deslauriers and S. Dubuc, Symmetric iterative interpolation, Constr. Approx. 5 (1989) 49-68.

[5] N. Dyn, Interpolation and approximation by radial and related functions, in: Approximation Theory, Vol. VI, eds. C.K. Chui, L.L. Schumaker and J. Ward (Academic Press, New York, 1989) pp. 211234.

[6] N. Dyn, Subdivision schemes in computer-aided geometric design, in: Wavelets, Subdivision Algorithms and Radial Basis Functions, Advances in Numerical Analysis, Vol. II, ed. W.A. Light (Oxford Univ. Press, Oxford, 1992) pp. 36-104.

[7] N. Dyn, J.A. Gregory and D. Levin, A four-point interpolatory subdivision scheme for curve design, Comput. Aided Geom. Design 4 (1987) 257-268.

[8] N. Dyn, D. Levin and J. Yoon, Non-stationary subdivision scheme by using radial basis functions interpolation, manuscript.

[9] W.R. Madych and S.A. Nelson, Multivariate interpolation and conditionally positive function, Approx. Theory Appl. 4(4) (1988) 77-89.

[10] W.R. Madych and S.A. Nelson, Multivariate interpolation and conditionally positive function II, Math. Comp. 54 (1990) 211-230.

[11] C.A. Micchelli, Interpolation of scattered data: Distances, matrices, and conditionally positive functions, Constr. Approx. 2 (1986) 11-22.

[12] M.J.D. Powell, The theory of radial basis functions approximation in 1990, in: Wavelets, Subdivision Algorithms and Radial Basis Functions, Advances in Numerical Analysis, Vol. II, ed. W.A. Light (Oxford Univ. Press, Oxford, 1992) pp. 105-210.

[13] Z. Wu and R. Schaback, Local error estimates for radial basis function interpolation of scattered data, IMA J. Numer. Anal. 13 (1993) 13-27.

[14] J. Yoon, Spectral approximation orders of radial basis function interpolation on the Sobolev space, SIAM J. Math. Anal. 33(4) (2001) 946-958.

[15] J. Yoon, $L_{p}$-error estimates for 'shifted' surface spline interpolation on Sobolev space, Math. Comp. 72(243) (2003) 1349-1367. 